\title{
Correlation between lattice distortion and friction stress in Ni-based
}

\section{equiatomic alloys}

\author{
Y.Y. Zhao and T.G. Nieh* \\ Department of Materials Science and Engineering, University of Tennessee, Knoxville, TN \\ 37996, USA \\ * Corresponding author. E-mail address: tnieh@utk.edu (T.G. Nieh).
}

\begin{abstract}
Many recent efforts have been made to apply traditional theories for solid solution strengthening to explain the strength increase in concentrated equiatomic alloys (or high-entropy alloys), but always faced the challenge of differentiating solvent from solute atoms. In this report, we conducted a systematical analysis of $\mathrm{Ni}$-based equiatomic alloys with a face-centered cubic structure and found that the lattice distortion in this alloy system could be simply described by the parameter of atomic size mismatch. It was found that lattice friction stresses of these alloys were well correlated with the lattice distortion. Dislocation core width in this Ni-based alloy system was also estimated and compared with that in the pure nickel. The intrinsically high strength in high-entropy alloys was probably resulted from a high lattice friction stress.
\end{abstract}

Keywords: High-entropy alloys; Yield strength; Lattice distortion; Lattice friction stress; Dislocation core width. 


\section{Introduction}

About a decade ago, Yeh proposed four 'core effects' in high-entropy alloys (HEAs, also known as multi-component concentrated alloys or equiatomic alloys): the high entropy effect, the lattice distortion effect, sluggish diffusion, and the 'cocktail' effect [1]. In the case of lattice distortion, a high-entropy alloy (HEA) consists of a number of constituent elements with different atomic sizes and electronic structures, so that its crystal lattice is expected to be distorted. In fact, several studies using x-ray and neutron have already demonstrated a decrease in diffraction peak intensity and broadening of peak width and were attributed to distorted lattice in HEAs [2-5]. However, it is difficult to physically visualize and quantitatively define lattice distortion at the atomic level. Some efforts have been made [6,7] to evaluate the distortion, but with only limited success. The usual approach is to statistically calculate the atomic size difference, but the physical picture how the lattice is distorted is still unclear [7]. Despite of the fact that lattice distortion is quantitatively ill defined, it is generally recognized [5] that lattice distortion is responsible for the observed unusual physical and mechanical properties of HEAs, such as strength enhancement.

For example, yield strength of the fully annealed pure Ni is only about $59 \mathrm{MPa}$ [8], which is much lower than that of the homogenized $[9,10]$ or fully recrystallized $[11,12]$, single-phase fcc (face-centered cubic) NiCoCrFeMn ( $200 \mathrm{MPa})$. The large strength difference triggered several research studies. These efforts primarily extended Fleischer's [13] solid-solution strengthening model for dilute alloys to concentrated alloys [14-16] with the attempt to reconcile the observed strength enhancement. However, an inevitable difficulty was encountered during the modeling because the impossibility to separate the solute from solvent atom in equiatomic concentrated alloys. Consequently, the applicability of the traditional solid-solution strengthening theory to 
these alloys becomes highly skeptical.

An alternative picture for the strengthening in HEAs is to imagine dislocations moving in a solution matrix that is uniformly (or globally) distorted by completely intermixed constituent atoms. In other words, one can envision the enhanced strength is simply a result of increased "intrinsic" or "friction" stress in the distorted lattice. In fact, Wu et al. [17] estimated the friction stress by calculating the Peierls stress, and concluded that the dislocation core should extend to about (1.0-1.5) $b$, where $b$ is the Burgers vector. It is also worthy of mentioning that several years ago, Yeh et al. [5] reported that the hardness as well as the lattice strain (or distortion) of equiatomic Ni-Al-Co-Cr-Cu-Fe-Si alloy systems was enhanced with increasing number of constituent elements. However, hardness value represents the overall strength of a polycrystal, which actually consists of several possible strengthening mechanisms, in addition to the lattice friction stress.

In this paper, we evaluate the intrinsic strength of pure $\mathrm{Ni}$ and a series of Ni-based fcc equiatomic binary, ternary, quaternary, and quinary solid-solution alloys from available data in literature. Meanwhile, lattice distortion in these equiatomic alloys are quantified using the lattice mismatch strain defined as the change of lattice parameter with respect to that of pure $\mathrm{Ni}$. We will present a semi-quantitative correlation to link the lattice friction stress with the lattice distortion and shed lights on the cause for the apparent high strength in equiatomic alloys.

\section{Results and discussion}

\subsection{Correlation between lattice distortion and yield strength}

Now, let us begin with the Cantor alloy, the equiatomic NiCoCrFeMn. This alloy has a singlephase fcc structure. Since Ni is the only constituent element in this alloy that has an fcc-structure, the Cantor alloy can be regarded as a Ni-based alloy, especially considering the lattice constant 
of NiCoCrFeMn $(a=0.3590 \mathrm{~nm}[18])$ is very close to that of $\mathrm{Ni}(a=0.3517 \mathrm{~nm}[8])$. Lattice distortion existing in the Cantor alloy can be envisioned in the following way. Pure fcc-Ni is considered to be the baseline reference state with every lattice site being occupied by the $\mathrm{Ni}$ atoms in the unit cell. Subsequently, Ni sites are gradually replaced by other constituent atoms according to the alloy stoichiometry and eventually the pure Ni evolves into the HEA. Upon atomic substitution, the bond length alters due to the atomic size mismatch and the difference in chemical specificity (e.g., electronegativity and charge transfer) between $\mathrm{Ni}$ and the substituted atom [19]. During the substitution procedure, the initial fec structure always remains and no second phase is formed. In this fashion, each substitution is anticipated to distort the fcc lattice and, after completion, the final lattice would be either dilated or contracted depending on the combined (average) effect of all substituted atoms. While the effect of chemical specificity is difficult to evaluate, let us assume it is minor in comparison to the atomic size effect. This is a reasonable assumption since, for the Cantor alloy $\mathrm{NiCoCrFeMn}$, the five constituent elements are immediately adjacent to each other in the Periodic Table.

The lattice distortion, to the first approximation, can be quantified using the volumetric distortion, $\Delta$, which is defined as $\Delta=\left(\Omega_{H E A}-\Omega_{N i}\right) / \Omega_{N i}$, where $\Omega_{H E A}$ and $\Omega_{N i}$ are the volume of unit cell of the HEA and Ni, respectively. The linear distortion, i.e., the lattice mismatch strain, $\delta$, is defined as $\delta=\left(a_{H E A}-a_{N i}\right) / a_{N i} \approx \Delta / 3$ [20], where $a_{H E A}$ and $a_{N i}$ are the lattice constant of the HEA and Ni, respectively. For simple discussion, we will use the lattice mismatch strain $\delta$ to describe the lattice distortion herein. Lattice constants and the $\delta$ values for pure $\mathrm{Ni}$ and a series of Ni-based fcc-equiatomic alloys, including those of two-components extending up to five-components, are listed in Table 1. 


\section{Table 1}

Lattice constant $(a)$, lattice distortion $(\delta)$, shear modulus $(G)$, grain size $(d)$, Poisson's ratio $(v), 0.2 \%$ offset yield strength $\left(\sigma_{y}\right)$, the lattice friction stress $\left(\sigma_{f}\right)$, corresponding $\sigma_{f} / G$, and the normalized dislocation core width $(w / b)$ of the equiatomic alloys investigated in this study.

\begin{tabular}{|c|c|c|c|c|c|c|c|c|c|c|}
\hline Alloys & $a(\AA)$ & $\delta$ & $G(\mathrm{GPa})$ & $d(\mu \mathrm{m})$ & $v$ & $\sigma_{y}(\mathrm{MPa})$ & $\sigma_{f}(\mathrm{MPa})$ & $\sigma_{f} / G\left(10^{-3}\right)$ & $w / b$ & Ref. \\
\hline $\mathrm{Ni}$ & 3.517 & 0 & 76 & - & 0.31 & 59 & 10 & 0.1 & 1.77 & [8] \\
\hline $\mathrm{NiCo}^{\mathrm{a}}$ & 3.535 & 0.0051 & 84 & 35 & 0.29 & 110 & 59 & 0.7 & 1.50 & {$[17,21]$} \\
\hline $\mathrm{NiFe}^{\mathrm{a}}$ & 3.583 & 0.0188 & 62 & 35 & 0.34 & 187 & 139 & 2.2 & 1.33 & {$[17,21]$} \\
\hline $\mathrm{NiCoMn}^{\mathrm{a}}$ & 3.598 & 0.0230 & 77 & 32 & 0.23 & 230 & 178 & 2.3 & 1.30 & {$[17,21]$} \\
\hline $\mathrm{NiFeMn}^{\mathrm{a}}$ & 3.616 & 0.0281 & 73 & 30 & 0.24 & 229 & 176 & 2.4 & 1.29 & {$[17,21]$} \\
\hline $\mathrm{NiCoCr}{ }^{\mathrm{a}}$ & 3.559 & 0.0119 & - & - & - & - & - & - & - & {$[17,21]$} \\
\hline $\mathrm{NiCoFe}^{\mathrm{a}}$ & 3.569 & 0.0148 & 60 & 28 & 0.35 & 213 & 161 & 2.7 & 1.30 & {$[17,21]$} \\
\hline $\mathrm{NiCoFe}^{\mathrm{b}}$ & 3.580 & 0.0179 & - & - & - & - & - & - & - & [22] \\
\hline $\mathrm{NiCoFe}^{\mathrm{b}}$ & 3.577 & 0.0171 & - & - & - & - & - & - & - & [23] \\
\hline $\mathrm{NiCoCrMn}^{\mathrm{a}}$ & 3.589 & 0.0205 & - & - & - & - & - & - & - & {$[17,21]$} \\
\hline $\mathrm{NiCoFeMn}^{\mathrm{a}}$ & 3.592 & 0.0213 & 77 & 48 & 0.22 & 176 & 131 & 1.7 & 1.34 & {$[17,21]$} \\
\hline $\mathrm{NiCoFeCr}^{\mathrm{a}}$ & 3.572 & 0.0156 & - & - & - & - & - & - & - & {$[17,21]$} \\
\hline $\mathrm{NiCoFeCr}^{\mathrm{b}}$ & 3.577 & 0.0171 & - & - & - & 140 & - & - & - & [24] \\
\hline $\mathrm{NiCoFeCr}^{\mathrm{c}}$ & 3.568 & 0.0145 & $84^{[17]}$ & 200 & $0.28^{[17]}$ & 130 & 101 & 1.2 & 1.41 & [24] \\
\hline $\mathrm{NiCoFeCr}^{\mathrm{b}}$ & 3.581 & 0.0182 & - & - & - & 147 & - & - & - & [25] \\
\hline $\mathrm{NiCoFeCr}{ }^{\mathrm{c}}$ & 3.578 & 0.0173 & $84^{[17]}$ & 290 & $0.28^{[17]}$ & 165 & 139 & 1.7 & 1.36 & {$[26]$} \\
\hline $\mathrm{NiCoCrFeMn}{ }^{a}$ & 3.599 & 0.0233 & 80 & 35 & 0.26 & 215 & 164 & 2.1 & 1.32 & {$[17,21]$} \\
\hline $\mathrm{NiCoCrFeMn}{ }^{b}$ & 3.602 & 0.0242 & - & - & - & 215 & - & - & - & [24] \\
\hline $\mathrm{NiCoCrFeMn}{ }^{c}$ & 3.595 & 0.0222 & $80^{[17]}$ & 130 & $0.26^{[17]}$ & 162 & 130 & 1.6 & 1.36 & [24] \\
\hline $\mathrm{NiCoCrFeMn}{ }^{\mathrm{b}}$ & 3.594 & 0.0219 & - & - & - & 209 & - & - & - & {$[9]$} \\
\hline$(\mathrm{NiCoCrFeMn})_{98} \mathrm{Al}_{2}{ }^{\mathrm{b}}$ & 3.615 & 0.0279 & - & - & - & - & - & - & - & [9] \\
\hline$(\mathrm{NiCoCrFeMn})_{96} \mathrm{Al}_{4}^{\mathrm{b}}$ & 3.619 & 0.0290 & - & - & - & 220 & - & - & - & [9] \\
\hline$(\mathrm{NiCoCrFeMn})_{93} \mathrm{Al}_{7}^{\mathrm{b}}$ & 3.631 & 0.0324 & - & - & - & 242 & - & - & - & [9] \\
\hline
\end{tabular}

${ }^{\text {a }}$ Samples prepared by arc melting, drop casting, homogenization, cold rolling and annealing.

${ }^{\mathrm{b}}$ Samples prepared by arc melting and, then, drop casting.

${ }^{\mathrm{c}}$ Samples prepared by arc melting, drop casting, and homogenization.

$0.2 \%$ offset yield strength $\left(\sigma_{y}\right)$ was determined at a strain rate of $1 \times 10^{-3} \mathrm{~s}^{-1}$ at room temperature. 


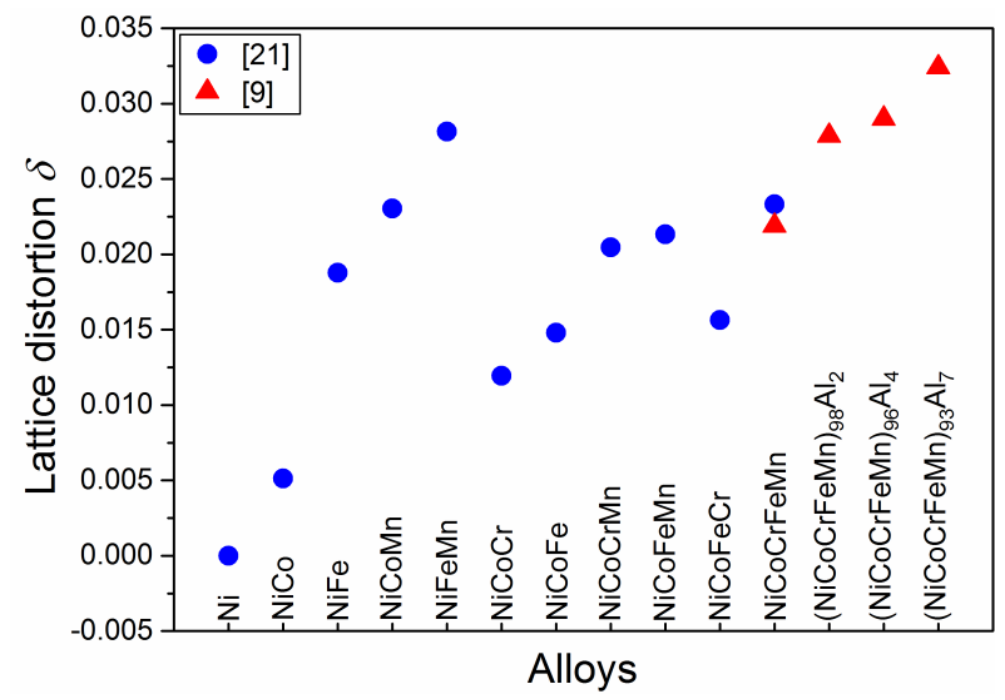

Fig. 1. Comparison of lattice distortion $(\delta)$ in the Ni-based fcc equiatomic alloys listed in Table 1.

A graph directly comparing lattice distortion of these Ni-based equiatomic alloys is displayed in Fig. 1. For convenience, the $\mathrm{x}$-axis is plotted in the order of increasing number of elements in alloys. It appears that more constituent elements can distort the lattice more. As mentioned before, Yeh et al. [5] also reported that lattice strain (or distortion) increased with the number of constituent elements. However, according to Fig. 1, it is not necessarily true per se. For instance, the Cantor alloy NiCoCrFeMn has the highest number of constituent elements in the equiatomic alloy series, but it is the 3-component NiFeMn that has the largest lattice distortion. In theory, adding another alloying element can cause either lattice expansion or contraction, depending on its relative atomic size and chemical bond with respect to its neighbors. It is pointed out that, among the five constituent elements of the Cantor alloy (NiCoCrFeMn), nickel atom is the smallest $\left(r_{N i} \sim 0.124 \mathrm{~nm}, r_{C o} \sim 0.125 \mathrm{~nm}, r_{F e} \sim 0.126 \mathrm{~nm}, r_{C r} \sim 0.128 \mathrm{~nm}\right.$ and $r_{M n} \sim 0.127 \mathrm{~nm}$ [27]). It is therefore a natural expectation that a higher-component alloy in this Ni-based HEA series would have a larger lattice constant. In fact, this concept extends even to higher alloying addition, for example, the largest distortion is found in the $(\mathrm{NiCoCrFeMn})_{100-x} \mathrm{Al}_{x}\left(\begin{array}{ll}x=0 & 7\end{array}\right)$ alloy system [9]. It is apparently caused by the substitution of larger $\mathrm{Al}$ atom $\left(r_{A l} \sim 0.143 \mathrm{~nm}\right.$ [27]). 


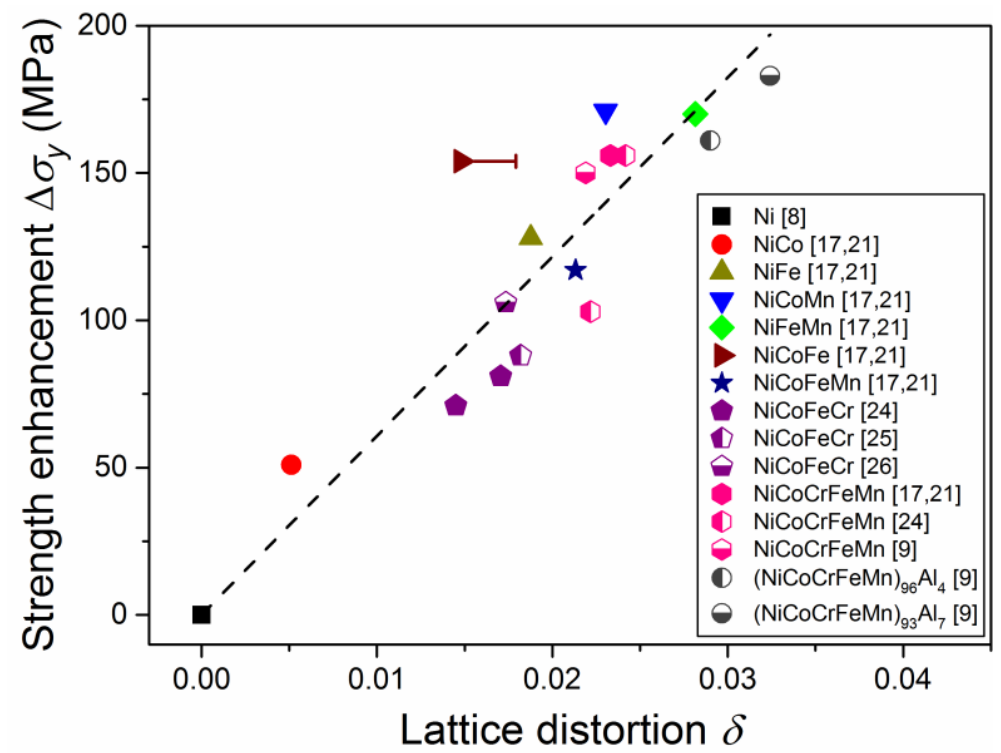

Fig. 2. Correlation between the strength enhancement $\left(\Delta \sigma_{y}\right)$ and lattice distortion $(\delta)$. The error bar of lattice distortion for NiCoFe is from Refs. [21-23].

Also included in Table 1 is the yield strength of the Ni-based equiatomic alloy series (from 2 to 5-components) in their fully annealed (or homogenized) states. Let us define strength enhancement $\left(\Delta \sigma_{y}\right)$ as the increase in yield strength with respective to pure nickel, that is, $\Delta \sigma_{y}=\sigma_{y, H E A}-\sigma_{y, N i}$, where $\sigma_{y, H E A}$ and $\sigma_{y, N i}$ are the yield strength of the HEA and pure $\mathrm{Ni}$, respectively. The correlation between $\Delta \sigma_{y}$ and $\delta$ is shown in Fig. 2. The graph clearly exhibits a general trend, namely, $\Delta \sigma_{y}$ monotonically increases with increasing $\delta$ in these Ni-based equiatomic alloys. The result appears to be similar to that reported in the equiatomic Ni-Al-Co$\mathrm{Cr}-\mathrm{Cu}-\mathrm{Fe}-\mathrm{Si}$ alloy system by Yeh et al. [5], but it needs to point out that Yeh's alloys are actually multi-phase (fcc+bcc, body-centered cubic) and, since the bcc phase is generally harder than fcc phase [28] and the amount of bcc phase increases with the number of constituent elements, their results can also be rationalized by the conventional composite theory.

\subsection{Correlation between lattice distortion and friction stress}

Plastic yielding is caused by dislocation motion in crystals. When dislocations move through a 
crystal lattice under stresses, they interact with various crystalline defects. In dilute solid-solution alloys, moving dislocations interact with surrounding point defects (solute atoms); this has been well described before [13]. In a HEA, on the other hand, even in the absence of crystalline defects, as long as all constituent atoms are equally partitioned, the interaction between a moving dislocation and distorted lattice is expected to be uniform throughout the entire lattice. In other words, the moving dislocation would experience a constant resistance (friction) and travel in a viscous manner through the distorted lattice. This is in an analogy to the lattice friction stress in a conventional alloy. To estimate this frictional stress, let us first evaluate the yield strength. The yield strength of a crystal $\sigma_{y}$ can be expressed as:

$$
\sigma_{y}=\sigma_{f}+\Delta \sigma_{s}
$$

where $\sigma_{f}$ is the lattice friction stress of the crystal and $\Delta \sigma_{s}$ includes contributions from all possible strengthening mechanisms, such as solid-solution hardening, dislocation hardening (or strain hardening), grain-boundary hardening, and precipitation hardening.

In the current work, precipitate is practically absent in the pure Ni or fully annealed Ni-based equiatomic alloys. Based on X-ray diffraction and scanning electron microscope analyses [17,29], these alloys are all single-phase fcc-alloys. Otto et al. [11] also confirmed that fully annealed equiatomic NiCoCrFeMn alloy is truly fcc single-phase without any precipitate. It is therefore reasonable to neglect precipitation hardening.

In terms of dislocation hardening (or Taylor hardening), the strength increase $\Delta \sigma_{d}$ can be estimated via equation [30]:

$$
\Delta \sigma_{d}=M \alpha G b \rho^{1 / 2}
$$

where $M=3.06$ is the Taylor factor for polycrystalline fcc-metals, $\alpha=0.2$ is an empirical 
constant [31], $G$ is the shear modulus, $b$ is the Burgers vector, and $\rho$ is the dislocation density. Since $\rho$ in the fully annealed pure $\mathrm{Ni}$ is on the order of $10^{12} \mathrm{~m}^{-2}[32,33]$, it is conceivable that $\rho$ in the fully annealed equiatomic alloys is also about $10^{12} \mathrm{~m}^{-2}$. Insert appropriate variables into Eq. (2), the strength increment $\Delta \sigma_{d}$ is estimated to be only about $12 \mathrm{MPa}$, which is relatively small compared to the yield strength of any of the equiatomic alloys listed in Table 1 . Therefore, a possible contribution from dislocation hardening can be ignored.

Another possible contribution is from grain-boundary hardening, $\Delta \sigma_{g b}$, which can be evaluated using the classical Hall-Petch relationship:

$$
\Delta \sigma_{g b}=k_{H P} d^{-1 / 2},
$$

where $d$ is the average grain size, and $k_{H P}$ is the Hall-Petch coefficient, which is $226 \mathrm{MPa} \cdot \mu \mathrm{m}^{1 / 2}$ for the fully annealed fcc-NiCoCrFeMn [34], slightly higher than the upper bound value for conventional fcc-alloys [28]. The value of $k_{H P}$ is not available for the majority of the multicomponent alloys listed in Table 1. However, it seems reasonable to apply $k_{H P}=226 \mathrm{MPa} \cdot \mu \mathrm{m}^{1 / 2}$ for the Ni-based equiatomic alloys in this study. Apply this $k_{H P}$ value, the strengthening from a grain size of $35 \mu \mathrm{m}$ is estimated to be about $38 \mathrm{MPa}$, which is not considered negligible but is still relatively small compared to the yield strength (>>100 MPa) of the listed Ni-based equiatomic alloys.

Traditional solid-solution strengthening theory describes the elastic interaction between solute atoms and moving dislocations as they move through the solvent lattice. As pointed out before, there is essentially no excess solute atom in an equiatomic "solid-solution" alloy, as long as the constituent atoms are completely intermixed. Therefore, there is, in principle, no contribution of solid-solution strengthening in any of the equiatomic alloys listed in Table 1, unless the alloy 
indeed contains dilute alloying addition. For instance, He et al. [9] studied single-phase fccNiCoCrFeMn alloyed with minor amounts of $\mathrm{Al}$, specifically $(\mathrm{NiCoCrFeMn})_{100-x} \mathrm{Al}_{\boldsymbol{x}}(\boldsymbol{x}=0-7)$, and observed solid-solution strengthening (substitutional). However, even in this study, the strengthening effect is still relatively weak ( 4 MPa per at.\% $\mathrm{Al})$.

In essence, $\Delta \sigma_{s}$ in Eq. (1), consists of, at the most, two terms: dislocation hardening and grain boundary hardening, with dislocation strengthening taking place within grain interior and grainboundary strengthening occurring at/in the vicinity of the boundaries (i.e. dislocation pile-ups). In other words, if the two mechanisms work collectively, the yield strength would be

$$
\sigma_{y}=\sigma_{f}+M \alpha G b \rho^{1 / 2}+k_{H P} d^{-1 / 2},
$$

or,

$$
\sigma_{f}=\sigma_{y}-M \alpha G b \rho^{1 / 2}-k_{H P} d^{-1 / 2}
$$

For the yield strength of fully annealed pure $\mathrm{Ni}$, we adopt data from the literature [35]:

$$
\sigma_{y, N i}=21.8[\mathrm{MPa}]+158\left[\mathrm{MPa} \cdot \mu \mathrm{m}^{1 / 2}\right] d^{-1 / 2}\left[\mu \mathrm{m}^{-1 / 2}\right]
$$

Insert it into Eq. (5), we obtain $\sigma_{f, N i}=10 \mathrm{MPa}$, where $\sigma_{f, N i}$ is the lattice friction stress of pure Ni. Using Eq. (5) we can further deduce the lattice friction stresses of the fully annealed (or homogenized) Ni-based equiatomic alloys and their values along with the corresponding $\sigma_{f} / G$ are summarized in Table 1. For clarity, we omit data from the $(\mathrm{NiCoCrFeMn})_{100-x} \mathrm{Al}_{x}(x=0 \quad 7)$ alloy system [9] since they contain solid-solution hardening part (although we have shown it is small).

We can now correlate the lattice friction stress as a function of lattice distortion and it is shown in Fig. 3. It is apparent that the lattice friction stress increases with the lattice distortion and, in fact, linearly scales with the lattice distortion. In other words, it is more difficult to move 
dislocations through an alloy matrix with larger lattice distortion. Similar to the $\Delta \sigma_{y}-\delta$ correlation (Fig. 2), ternary $\mathrm{NiCoMn}$ and $\mathrm{NiFeMn}$ have the largest lattice distortion, thus the highest friction stresses in the present Ni-based equiatomic alloy systems.

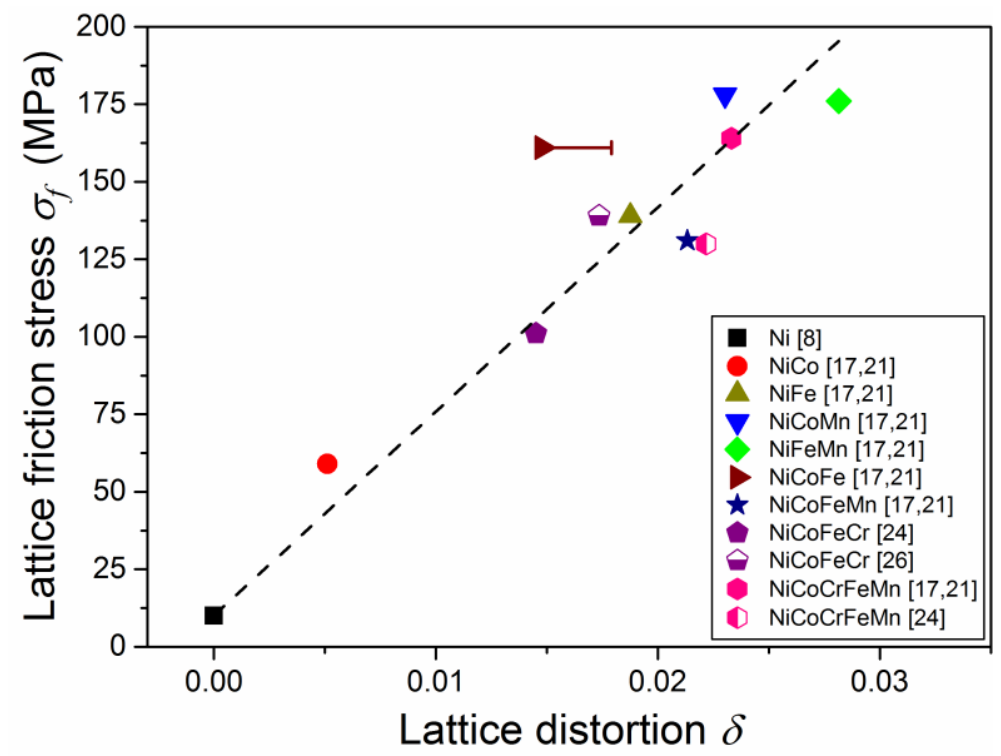

Fig. 3. Correlation between the lattice friction stress $\left(\sigma_{f}\right)$ and the lattice distortion $(\delta)$. The error bar of lattice distortion for $\mathrm{NiCoFe}$ is from Refs. [21-23].

According to Fig. 3, the average value of the lattice friction stress for multicomponent $(\geq 3)$ equiatomic alloys is about $130-170 \mathrm{MPa}\left(\sim 2 \times 10^{-3} G\right)$, which is about one to two orders of magnitude higher than that for the conventional fcc-metals, which is about $10^{-5} G-10^{-4} G$ [36]. However, it is still far less than that for the conventional bcc-metals $\left(\sim 10^{-2} G[36]\right)$. This is consistent with the notion that yielding in the current fcc-Ni-based equiatomic alloys is more difficult than that in the traditional fcc-alloys.

The correlation between lattice friction stress and lattice distortion is rationalized in the following way. Lattice friction results from spatial perturbation in interaction between the dislocation and the lattice, which usually exhibits itself in a periodic manner and is independent of crystalline defects. The current definition of lattice distortion parameter $\delta$ represents only the average expansion of the overall lattice. At the atomic scale, the location of each atom is actually 
affected by its nearest neighbors, as noted by Guo et al [4]. In other words, the fcc unit cell is no longer an equilateral cube, like the conventional one, but becomes irregular. The irregularity or shape of the cell is determined by the combined effect of the nearest atomic size mismatch and chemical bond. In such case, the dislocation line is not straight, but wiggled consisting of successive segments, as illustrated in Fig. 4. Total energy of the dislocation is

$$
E_{d}=G_{i} \quad b_{i}^{2} l_{i}
$$

where $l_{i}$ is the adjacent atomic distance, $G$ is the shear modulus, and $b_{i}$ is Burgers vector. It is still unclear about the exact value of $E_{d}$ because the uncertainty of $l_{i}$. However, based on the fact that $\delta$ is positive, i.e., lattice dilation, the energy of this dislocation "line" is expected to be higher than that of a perfectly straight dislocation. The energy and stress required to move such a dislocation are also expected to be higher. Quantitative evaluation of the effect of wiggled dislocation is currently not possible unless we know exactly the interatomic bonding energies between constituent atoms, which determine the bond length (or interatomic distance). Atomistic simulation may shed lights, but it would be beyond the scope of the current paper.

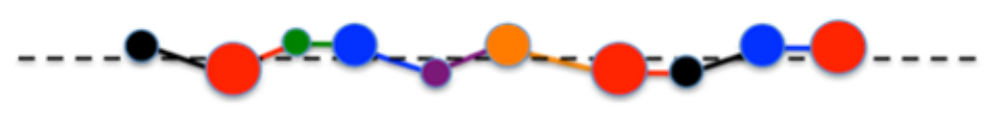

Fig. 4. The schematic of the wiggled dislocation line in the distorted HEA lattice.

Another contributing factor is the possible narrowing of dislocation core. The classical theory for lattice friction stress (or Peierls stress) is described by the equation [37,38]:

$$
\tau_{f}=\frac{2 G}{1-v} \exp \left[\frac{-2 \pi w}{b}\right]
$$

where $\tau_{f}$ is the lattice friction stress (shear), $G$ the shear modulus, $v$ the Poisson's ratio, $w$ the width of dislocation core, and $b$ the Burgers vector. The dislocation core width normalized by 
the Burgers vector is

$$
\frac{w}{b}=\frac{1}{2} \ln \left[\frac{2 M G}{{ }_{f}(1)}\right],
$$

where $\sigma_{f}=M \tau_{f}$, and $M$ is the Taylor factor. Insert each parameter, the values of $w / b$ for the Ni-based equiatomic alloys are listed in Table 1 and also plotted in Fig. 5. In comparison, pure Ni has a much higher $w / b$ value $(w / b=1.77)$ than the Ni-based equiatomic alloys $(w / b<1.50)$, again indicating more difficult dislocation glide in the equiatomic fec alloys. Also noted in Fig. 5 is that, except for the binary $\mathrm{NiCo}, w / b$ values for the Ni-based equiatomic alloys fall within the range of $1.30-1.40$ and are nearly constant ( $w / b=1.33 \pm 0.04)$, indicating that the lattice friction stress in these equiatomic alloys approximately scales linearly with $G /(1-v)$.

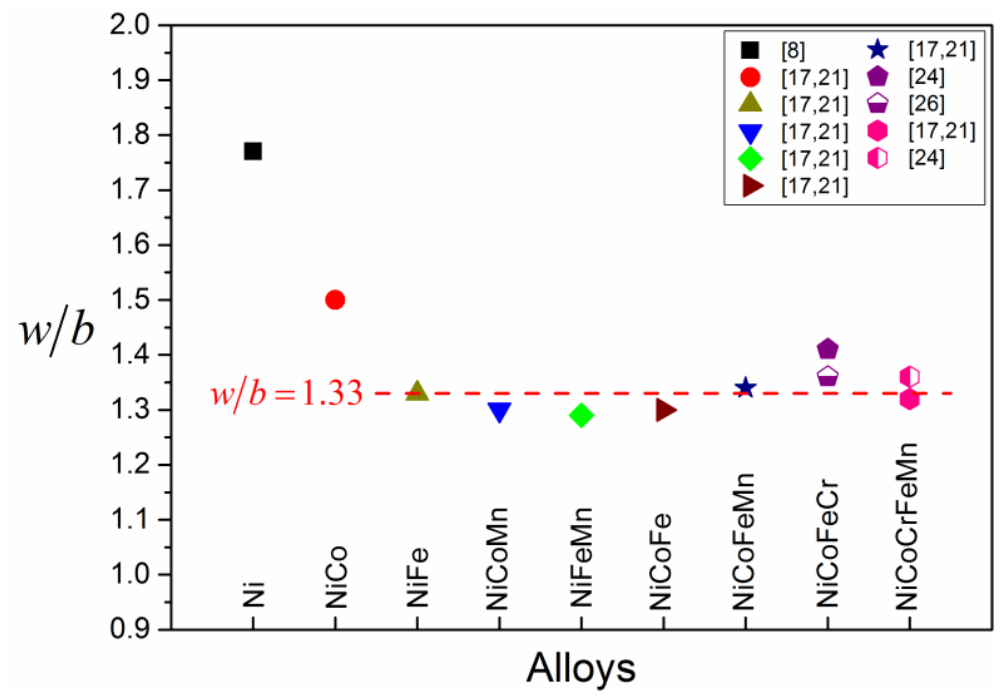

Fig. 5. The normalized width of dislocation core $(w / b)$ in pure nickel and Ni-based equiatomic alloys listed in Table 1.

The above lattice friction-lattice distortion relationship prevails in fcc-HEAs. For HEAs with other structures, such as bcc and hexagonal close-packed (hcp), we anticipate to have a similar result. In fact, remarkable enhancement in yield strength has also been observed in these alloys [14,39-42]. However, there are insufficient data allowing for a thorough analysis at the present 
time.

\section{Conclusions}

We have carried out a systematic analysis of the lattice distortion in a series of Ni-based equiatomic alloys. The lattice distortion is not necessarily increased monotonically with the number of constituent elements, but also depends on the alloy chemistry. By taking into account all possible strengthening mechanisms, the lattice friction stresses in the current Ni-based alloy system were deduced and found to scale linearly with the lattice distortion. Additionally, the width of dislocation core in these Ni-based equiatomic alloys was estimated to be about $1.33 b$, where $b$ is the Burgers vector, falling below the value reported for pure fcc-Ni. The higher friction stress in multicomponent equiatomic alloys may, therefore, be attributable to the wiggled dislocation line and a smaller dislocation core. The above finding suggests that the generally observed high strength in equiatomic alloys is probably not attributed to the traditional solid solution strengthening as suggested in several recent publications.

\section{Acknowledgements}

This work is supported by NSF under contract DMR-1408722.

\section{References}

[1] J.W. Yeh, Recent progress in high entropy alloys, Ann. Chim. Sci. Mat. 31 (2006) 633-648.

[2] J.W. Yeh, S.J. Lin, T.S. Chin, J.Y. Gan, S.K. Chen, T.T. Shun, C.H. Tsau, S.Y. Chou, Formation of simple crystal structures in $\mathrm{Cu}-\mathrm{Co}-\mathrm{Ni}-\mathrm{Cr}-\mathrm{Al}-\mathrm{Fe}-\mathrm{Ti}-\mathrm{V}$ alloys with multiprincipal metallic elements, Metall. Mater. Trans. A 35 (2004) 2533-2536.

[3] B.S. Murty, J.W. Yeh, S. Ranganathan, High-Entropy Alloys, Elsevier, Amsterdam, 2014.

[4] W. Guo, W. Dmowski, J.Y. Noh, P. Rack, P.K. Liaw, T. Egami, Local atomic structure of a high-entropy alloy: an X-ray and neutron scattering study, Metall. Mater. Trans. A 44 (2013) 1994-1997.

[5] J.W. Yeh, S.Y. Chang, Y.D. Hong, S.K. Chen, S.J. Lin, Anomalous decrease in X-ray diffraction intensities of $\mathrm{Cu}-\mathrm{Ni}-\mathrm{Al}-\mathrm{Co}-\mathrm{Cr}-\mathrm{Fe}-\mathrm{Si}$ alloy systems with multi-principal elements, Mater. Chem. Phys. 103 (2007) 41-46.

[6] S. Wang, Atomic structure modeling of multi-principal-element alloys by the principle of maximum entropy, Entropy 15 (2013) 5536-5548.

[7] Y. Zou, S. Maiti, W. Steurer, R. Spolenak, Size-dependent plasticity in an $\mathrm{Nb}_{25} \mathrm{Mo}_{25} \mathrm{Ta}_{25} \mathrm{~W}_{25}$ refractory highentropy alloy, Acta Mater. 65 (2014) 85-97. 
[8] ASM Handbook Vol. 2, Properties and Selection: Nonferrous Alloys and Special-Purpose Materials, ASM International, Materials Park, OH, 1990.

[9] J.Y. He, W.H. Liu, H. Wang, Y. Wu, X.J. Liu, T.G. Nieh, Z.P. Lu, Effects of Al addition on structural evolution and tensile properties of the FeCoNiCrMn high-entropy alloy system, Acta Mater. 62 (2014) 105-113.

[10] Y. Wu, W.H. Liu, X.L. Wang, D. Ma, A.D. Stoica, T.G. Nieh, Z.B. He, Z.P. Lu, In-situ neutron diffraction study of deformation behavior of a multi-component high-entropy alloy, Appl. Phys. Lett. 104 (2014) 051910.

[11] F. Otto, A. Dlouhý, Ch. Somsen, H. Bei, G. Eggeler, E.P. George, The influences of temperature and microstructure on the tensile properties of a CoCrFeMnNi high-entropy alloy, Acta Mater. 61 (2013) 57435755 .

[12] A. Gali, E.P. George, Tensile properties of high- and medium-entropy alloys, Intermetallics 39 (2013) 74-78.

[13] R.L. Fleischer, Substitutional solution hardening, Acta Metall. 11 (1963) 203-209.

[14] O.N. Senkov, J.M. Scott, S.V. Senkova, D.B. Miracle, C.F. Woodward, Microstructure and room temperature properties of a high-entropy TaNbHfZrTi alloy, J. Alloys Compd. 509 (2011) 6043-6048.

[15] I. Toda-Caraballo, P.E. Rivera-Díaz-del-Castillo, Modelling solid solution hardening in high entropy alloys, Acta Mater. 85 (2015) 14-23.

[16] C. Varvenne, G. Leyson, M. Ghazisaeidi, W. Curtin, Solute strengthening in random alloys, Acta Mater. (2016). http://dx.doi.org/10.1016/ j.actamat.2016.09.046

[17] Z. Wu, H. Bei, G.M. Pharr, E.P. George, Temperature dependence of the mechanical properties of equiatomic solid solution alloys with face-centered cubic crystal structures, Acta Mater. 81 (2014) 428-441.

[18] B. Cantor, I.T.H. Chang, P. Knight, A.J.B. Vincent, Microstructural development in equiatomic multicomponent alloys, Mater. Sci. Eng. A 375 (2004) 213-218.

[19] T. Egami, M. Ojha, O. Khorgolkhuu, D.M. Nicholson, G.M. Stocks, Local electronic effects and irradiation resistance in high-entropy alloys, JOM 67 (2015) 2345-2349.

[20] R.C. Reed, The Superalloys: Fundamentals and Applications, Cambridge university press, Cambridge, UK, 2006.

[21] Z. Wu, Temperature and Alloying Effects on the Mechanical Properties of Equiatomic FCC Solid Solution Alloys, PhD diss., University of Tennessee, 2014. http://trace.tennessee.edu/utk_graddiss/2884

[22] S. Guo, C. Ng, Z. Wang, C.T. Liu, Solid solutioning in equiatomic alloys: limit set by topological instability, J. Alloys Compd. 583 (2014) 410-413.

[23] X. Wang, H. Xie, L. Jia, Z.L. Lu, Effect of Ti, $\mathrm{Al}$ and $\mathrm{Cu}$ addition on structural evolution and phase constitution of FeCoNi system equimolar alloys, Mater. Sci. Forum 724 (2012) 335-338.

[24] G.A. Salishchev, M.A. Tikhonovsky, D.G. Shaysultanov, N.D. Stepanov, A.V. Kuznetsov, I.V. Kolodiy, A.S. Tortika, O.N. Senkov, Effect of Mn and V on structure and mechanical properties of high-entropy alloys based on CoCrFeNi system, J. Alloys Compd. 591 (2014) 11-21.

[25] W.H. Liu, J.Y. He, H.L. Huang, H. Wang, Z.P. Lu, C.T. Liu, Effects of Nb additions on the microstructure and mechanical property of CoCrFeNi high-entropy alloys, Intermetallics 60 (2015) 1-8.

[26] J.Y. He, H. Wang, H.L. Huang, X.D. Xu, M.W. Chen, Y. Wu, X.J. Liu, T.G. Nieh, K. An, Z.P. Lu, A precipitation-hardened high-entropy alloy with outstanding tensile properties, Acta Mater. 102 (2016) 187-196.

[27] N.N. Greenwood, A. Earnshaw, Chemistry of the Elements, Pergamon Press, Oxford, UK, 1984.

[28] D. Wu, J. Zhang, J. Huang, H. Bei, T.G. Nieh, Grain-boundary strengthening in nanocrystalline chromium and the Hall-Petch coefficient of body-centered cubic metals, Scripta Mater. 68 (2013) 118-121.

[29] Z. Wu, H. Bei, F. Otto, G.M. Pharr, E.P. George, Recovery, recrystallization, grain growth and phase stability of a family of FCC-structured multi-component equiatomic solid solution alloys, Intermetallics 46 (2014) 131140.

[30] J.E. Bailey, P.B. Hirsch, The dislocation distribution, flow stress, and stored energy in cold-worked polycrystalline silver, Philos. Mag. 5 (1960) 485-497.

[31] T.H. Courtney, Mechanical Behavior of Materials, Waveland Press, Long Grove, IL, 2000.

[32] T. Narutani, J. Takamura, Grain-size strengthening in terms of dislocation density measured by resistivity, Acta Metall. Mater. 39 (1991) 2037-2049.

[33] G.K. Williamson, R.E. Smallman, III. Dislocation densities in some annealed and cold-worked metals from measurements on the X-ray debye-scherrer spectrum, Philos. Mag. 1 (1956) 34-46.

[34] W.H. Liu, Y. Wu, J.Y. He, T.G. Nieh, Z.P. Lu, Grain growth and the Hall-Petch relationship in a high-entropy FeCrNiCoMn alloy, Scripta Mater. 68 (2013) 526-529.

[35] A.A. Thompson, Yielding in nickel as a function of grain or cell size, Acta Metall. 23 (1975) 1337-1342.

[36] Y. Kamimura, K. Edagawa, S. Takeuchi, Experimental evaluation of the Peierls stresses in a variety of crystals and their relation to the crystal structure, Acta Mater. 61 (2013) 294-309. 
[37] R. Peierls, The size of a dislocation, Proc. Phys. Soc. 52 (1940) 34-37.

[38] F.R.N. Nabarro, Dislocations in a simple cubic lattice, Proc. Phys. Soc. 59 (1947) 256-272.

[39] O.N. Senkov, G.B. Wilks, J.M. Scott, D.B. Miracle, Mechanical properties of $\mathrm{Nb}_{25} \mathrm{Mo}_{25} \mathrm{Ta}_{25} \mathrm{~W}_{25}$ and $\mathrm{V}_{20} \mathrm{Nb}_{20} \mathrm{Mo}_{20} \mathrm{Ta}_{20} \mathrm{~W}_{20}$ refractory high entropy alloys, Intermetallics 19 (2011) 698-706.

[40] O.N. Senkov, G.B. Wilks, D.B. Miracle, C.P. Chuang, P.K. Liaw, Refractory high-entropy alloys, Intermetallics 18 (2010) 1758-1765.

[41] K.M. Youssef, A.J. Zaddach, C. Niu, D.L. Irving, C.C. Koch, A novel low-density, high-hardness, highentropy alloy with close-packed single-phase nanocrystalline structures, Mater. Res. Lett. 3 (2015) 95-99.

[42] L. Rogal, F. Czerwinski, P.T. Jochym, L. Litynska-Dobrzynska, Microstructure and mechanical properties of the novel $\mathrm{Hf}_{25} \mathrm{Sc}_{25} \mathrm{Ti}_{25} \mathrm{Zr}_{25}$ equiatomic alloy with hexagonal solid solutions, Mater. Des. 92 (2016) 8-17. 

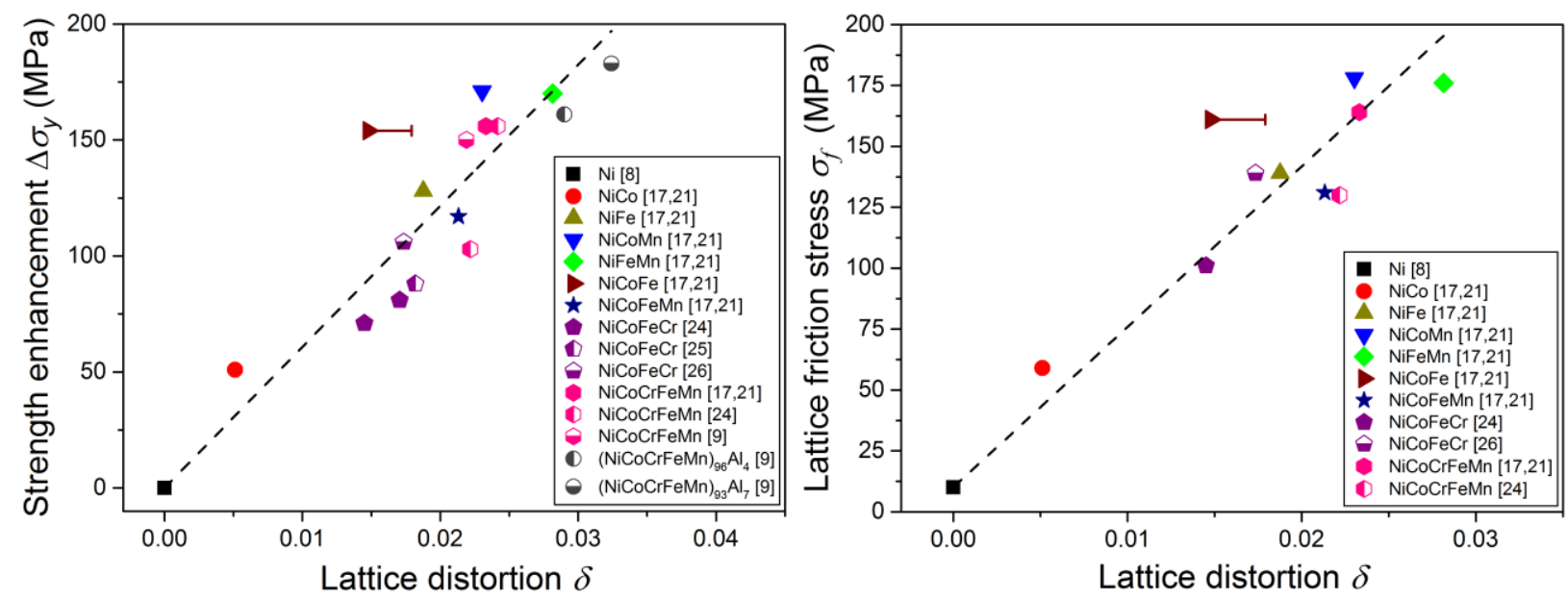